\title{
Enhanced circulating PCSK9 concentration by berberine through SREBP-2 pathway in high fat diet-fed rats
}

Yan-Jun Jia, Rui-Xa Xu, Jing Sun, Yue Tang ${ }^{*}$ and Jian-Jun Li ${ }^{*}$

\begin{abstract}
Background: Berberine (BBR), a natural plant extract, has been shown to improve lipid metabolism. However, its effects on PCSK9, a key factor involving in the lipid metabolism, have not yet been evaluated in vivo. The aim of the present study was to investigate the effect of BBR on PCSK9 expression in high fat diet-fed (HFD) rats.

Methods: Thirty-two male Sprague Dawley (SD) rats were randomized into the four groups $(n=8)$ : normal diet (Control), HFD, HFD + simvastatin (Sim, $2 \mathrm{mg} / \mathrm{kg} / \mathrm{d}$ ) and HDF + BBR (400 mg/kg/d) for 6 weeks. The following parameters were determined: 1) body weight; 2) serum lipid profile; 3) serum PCSK9 measured by enzyme-linked immuno sorbent assay (ELISA) ; 4) hepatic expressions of low-density lipoprotein receptor (LDLR), sterol regulatory element binding protein-2 (SREBP-2) and hepatocyte nuclear factor 1 (HNF1) were examined by real time quantitative polymerase chain reaction (RT-PCR) and western blotting analysis.

Results: Compared with HFD rats, Sim and BBR significantly reduced body weight gain and improved lipid profile $(P<0.05$ respectively). In addition, either of drug treatment for 6 weeks could increase serum concentration of PCSK9 in HFD rats $(P<0.05)$. This enhanced PCSK9 expression was demonstrated to be associated with the up-regulation of hepatic expression of LDLR and SREBP-2 and the down-regulation of hepatic expression of HNF1 ( $P<0.05$ respectively).

Conclusions: The data provided the first line of the evidence that BBR, similar to the Sim, could increase the expression of PCSK9 levels in HFD rats through SREBP-2 activation, suggesting that impacts of BBR on lipid profile may also be linked to SREBP-2 pathway.
\end{abstract}

Keywords: Berberine, Simvastatin, Proprotein convertase subtilisin/kexin type 9, Low density lipoprotein receptor, High fat diet-fed rats

\section{Introduction}

It has been well established that high total cholesterol (TC) and low density lipoprotein cholesterol (LDL-C) are among the most important predictors of future coronary artery disease (CAD) and cardiovascular events [1]. In contrast, reduction in the circulating levels of TC and LDL-C has been demonstrated to significantly reduce the risk for CAD [2]. Therefore, the effective control of dyslipidemia and better understanding of lipid metabolism have been considered as a powerful strategy for the prevention and treatment of CAD [3].

\footnotetext{
* Correspondence: Tangyue1226@vip. sina.com; lijianjun938@yahoo.com Division of Dyslipidemia, State Key Laboratory of Cardiovascular Disease, Fu Wai Hospital, National Center for Cardiovascular Disease, Chinese Academy of Medical Sciences and Peking Union Medical College, Beijing 100037,
} China

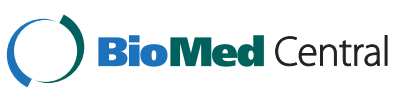

(c) 2014 Jia et al.; licensee BioMed Central Ltd. This is an Open Access article distributed under the terms of the Creative Commons Attribution License (http://creativecommons.org/licenses/by/2.0), which permits unrestricted use, distribution, and reproduction in any medium, provided the original work is properly credited. The Creative Commons Public Domain Dedication waiver (http://creativecommons.org/publicdomain/zero/1.0/) applies to the data made available in this article unless otherwise stated.
Recent studies of human genetics and genome-wide screens have identified proprotein convertase subtilisin/ kexin type 9 (PCSK9) as the third gene associated with autosomal dominant familial hypercholesterolemia, after LDLR and apoB100 [4]. The function of PCSK9, as a secreted serine protease, is degradation of hepatic LDLR, which is directly correlates with its tight association with plasma cholesterol levels and provides a new therapeutic target to combat hypercholesterolemia and CAD [5-8]. At the transcriptional level, PCSK9 has been identified as a target gene of SREBPs [9-11]. The proximal promoter of the PCSK9 gene contains a functional sterol regulatory element (SRE) that responds to changes in intracellular cholesterol levels [12]. In HepG2 cells both SREBP-1 and SREBP-2 transcriptionally activate PCSK9 
via this SRE site [9]. In vivo however, it was suggested that the sterol-dependent regulation of PCSK9 is mediated predominantly by SREBP-2 [11].

The most common used lipid-lowering drugs currently is the statins including China. These drugs act as competitive inhibitors of 3-hydroxy-3-methylglutarylcoenzyme A (HMG CoA) reductase, leading to the reduction of endogenous cholesterol synthesis, depletion of intracellular cholesterol levels and a subsequent up-regulation of the low-density lipoprotein receptors (LDLRs) through the sterol regulatory element binding protein (SREBP) pathway [3,4]. In addition, an increased hepatic LDLR expression can result in the improvement in clearance of plasma LDL-C [5-11].

Recently, statins have also been shown to enhance the expression of PCSK9 gene through the SREBP pathway [12]. Several studies have reported that statin treatment not only decreases LDL-C and improves cardiovascular adverse events but also causes an increase in the concentration of serum PCSK9 [13-17]. Moreover, it was postulated that statins increased the activity/nuclear translocation of SREBP-2, resulting in the increased expression and secretion of the PCSK9 protein. Meanwhile, several other studies have also indicated that another commonly prescribed lipid-lowering agent, fenofibrate, is also reported to increase the circulating levels of PCSK9 $[18,19]$.

Berberine (BBR) is an isoquinoline plant alkaloid and has long been used in Chinese medicine [20-23]. BBR has commonly been used as a traditional herbal medicine to treat bacterial infection and many other illnesses without toxic effects reported to date in a body of preclinical and clinical studies [20,21]. Interestingly, BBR has also been demonstrated to lower fasting triglyceride (TG) levels in a clinical trial and reduce body weight as well as improve dyslipidemia in high fat diet-fed (HFD) rats. Hence, the understanding the cardiovascular protective effects of BBR appears to be mounting [22,23].

The aim of the present study, therefore, was to evaluate the effects of BBR on plasma PCSK9 concentration as well as its potential mechanism using HFD rat model.

\section{Methods}

\section{Drugs and regents}

BBR Chloride was purchased from haoran biological technology company (shanghai city, China). Simvastatin (Sim) was kindly provided by Merck Research Laboratories (Hangzhou city, China). BBR or SS were dissolved in $0.5 \%$ sodium chloride before use.

\section{Animal studies}

After weaning, 3-week male Sprague-Dawley (SD) rats (average body weight, $50 \pm 1 \mathrm{~g}$ ) were purchased from animal center of Beijing University Health Science Center.
All animals were placed in cage (four rats per cage) in an atmosphere of $55 \pm 10 \%$ relative humidity at $22 \pm 2^{\circ} \mathrm{C}$ with a $12 \mathrm{~h} \mathrm{light/dark} \mathrm{cycle.} \mathrm{Rats} \mathrm{were} \mathrm{given} \mathrm{free} \mathrm{access} \mathrm{to} \mathrm{food}$ and water. The study protocol was approved by the ethics committee on Animal Center of Beijing University Health Science.

After adapting to the environment for a week, animals were randomly assigned into the four groups $(\mathrm{n}=8$ for each group): Control (Con) group, HFD group, HFD + Sim group, HFD + BBR group. The indicating diets were purchased from Beijing keao Feed Company. Rat in Con group was fed with a standard chow diet $(3.21 \mathrm{kcal} / \mathrm{g}$, $13 \%$ calories from fat, $25 \%$ calories from protein, $62 \%$ calories from carbohydrate) for 6 weeks. The HFD group was fed with a HFD $(4.39 \mathrm{kcal} / \mathrm{g}, 47 \%$ calories from fat, $20 \%$ calories from protein, $33 \%$ calories from carbohydrate) for 6 weeks. The HFD + BBR group was fed with a HFD plus BBR (400 mg/kg/d, oral input) for 6 weeks. The HFD + Sim group was fed with a HFD plus Sim ( $2 \mathrm{mg} / \mathrm{kg} / \mathrm{d}$, oral input) for 6 weeks. During experiments, rats had free access to water and food and food consumption was measured every day for the purpose of excluding the changes of body weight or lipid profile due to the variation of caloric intake. At the end of the experiment, all animals were anesthetized with ether and the blood samples were collected in EDTA-coated tubes and centrifuged. The plasma and livers were rapidly collected and stored at $-80^{\circ} \mathrm{C}$ until analysis.

\section{Body weight measurement and biochemical analysis}

Animal's body weight was measured in every week from week 0 (before treatment) to week 6 (the end of the study).

Fasting blood samples were collected and plasma levels of TC, TG, high density lipoprotein cholesterol (HDL-C) were determined on an automatic biochemistry analyzer (Hitachi 7150, Tokyo, Japan). The LDL-C was estimated according to the following formula: LDL-C $=$ TC-HDL-C-TG/5.

\section{Assay of plasma PCSK9 concentration}

PCSK9 concentrations were measured using a highsensitivity, quantitative sandwich enzyme immunoassay (Quantikine ELISA, R\&D systems Europe Ltd, Sweden) according to our previous publications $[14,15]$. The lower limit of detection was $0.096 \mathrm{ng} / \mathrm{ml}$. According to the manufacturer, the inter-assay coefficient of variation is less than $5 \%$.

\section{Detection of hepatic LDLR, SREBP-2 and hepatocyte nuclear factor-1 (HNF-1) mRNA expression}

Key enzymes of lipid metabolism, which were analyzed by real time quantitative polymerase chain reaction (RT-PCR), were selected as candidate genes for the assessment of their mRNA expression levels in the liver 
of rats studied (Table 1). Briefly, total RNA in liver tissue was isolated from hepatic tissue using the Trizol reagent Kit (Invitrogen, USA) following the manufacture's instructions. And then, it was measured by spectrophotometry at an absorbance of $260 \mathrm{~nm}$, and designated the purity valid if the ratio of A260/A280 was in the range from 1.8 to 2 . The integrity of the RNA was checked by denaturing agarose gel electrophoresis and ethidium bromide staining. $3 \mu \mathrm{g}$ of the total RNA was reversed transcribed by reverse Aid First Strand cDNA synthesis kit (Fermentas, CA, USA). The abundances of key genes (LDLR, SREBP-2 and HNF-1) and glyceraldehyde-3phosphate dehydrogenase (GAPDH) mRNA were analyzed by RT-PCR using the 7500HT RT-PCR system (Applied Biosystems, Foster, CA, USA). RT-PCR was performed using the SYBR Premix ExTaq (TaKaRa Bio Inc. Japan) according to the manufacturer's instructions.

Standard curves for each primer pair were generated by serial dilutions of cDNA from a reference sample and used for regression analyses. All PCR assays were performed in triplicate. The variance of the triplicate measurements was $<1 \%$. Results were analyzed using the standard curve method by the sequence detection systems (SDS) software. The data was expressed as the relative levels of mRNA after normalized with GAPDH.

\section{Western blot analysis of LDLR, SREBP-2 protein expression}

Western blot analysis was performed to examine the protein expression in the liver tissue of rats studied. Briefly, the liver sample $(0.2 \mathrm{~g})$ were homogenized in $3 \mathrm{ml}$ of lysing buffer (25 mM MES, pH 6.5, $0.15 \mathrm{M} \mathrm{NaCl}$, $1 \% \mathrm{v} / \mathrm{v}$ Triton X-100, $60 \mathrm{mM}$ octylglucoside, and $1 \mathrm{X}$ protein inhibitors) using a Dounce homogenizer. After incubation on ice for $20 \mathrm{~min}$, homogenates were centrifuged at $12,000 \mathrm{~g}$ for $15 \mathrm{~min}$ at $4^{\circ} \mathrm{C}$. Supernatants were collected and protein concentration was measured using

Table 1 The Sequences of primers for Real-Time PCR used in the study

\begin{tabular}{lll}
\hline Gene description & Primer & Sequences $\left(\mathbf{5}^{\prime} \boldsymbol{\rightarrow} \mathbf{3}^{\prime}\right)$ \\
\hline LDLR & $\mathbf{F}$ & GATTGGCTATGAGTGCCTATGTC \\
SREBP-2 & $\mathbf{R}$ & GTGAAGAGCAGAAACCCTATGG \\
& $\mathbf{F}$ & AGCATACCGCAAGGTGTTCC \\
HNF1 & $\mathbf{R}$ & CCAGGTGTCTACTTCTCCGTGT \\
& $\mathbf{F}$ & ATGACACGGATGACGATGGG \\
GAPDH & $\mathbf{R}$ & ATGGGTCCTCCTGAAGAAGTGA \\
& $\mathbf{F}$ & ACAGCAACAGGGTGTGGAC \\
& $\mathbf{R}$ & TTGAGGGTGCAGCGAACT
\end{tabular}

$\mathrm{PCR}=$ polymerase chain reaction; $\mathrm{F}=$ forward; $\mathrm{R}=$ reverse; $\mathrm{LDLR}=$ low density lipoprotein receptor; $\mathrm{PCSK} 9=$ proprotein convertase subtilisin/kexin type 9; SREBP-2 = sterol regulatory element binding protein-2; HNF1 = Hepatocyte Nuclear Factor 1; GAPDH = reduced glyceraldehyde-phosphate dehydrogenase. a BCA protein detection kit. We separated the proteins on $10 \%$ SDS polyacrylamide gels under reducing conditions and transferred them, eletrophoretically, onto PVDF membranes (Bio-Rad, USA). Subsequently, membranes were blocked in $10 \%$ bovine serum albumin in Tris buffered saline with $0.1 \%$ Tween 20 for 30 minutes. Membranes were incubated with primary antibody (anti-LDLR antibody, anti-SREBP- 2 antibody and $\beta$-Actin antibody) at $4^{\circ} \mathrm{C}$ for overnight. After three washings with Tris buffered saline containing 0.1\% Tween 20 for 15 minutes, membranes were incubated with second antibody at a 1:1000 dilution for 30 minutes. After washing, the membranes were developed with ECL western blotting reagents according to the manufacturer's instructions. Films were scanned and quantified by using ImageQuant software (Molecular Dynamics).

\section{Statistical analysis}

Results are expressed as the means \pm standard deviation (SD). Statistical analysis was performed using SPSS 19.0 statistical packages (Chicago, Illinois, USA). The significance of differences was evaluated by a one-way analysis of variance (ANOVA) for unpaired data. The significance level was chosen as $\mathrm{P}<0.05$.

\section{Results}

Changes of body weight and plasma lipid levels induced by Sim and BBR

Rats fed with HFD tended to developing obesity, shown by increased body weight compared with controls, while either Sim or BBR could decrease the body weight of rats fed with HFD $(\mathrm{P}<0.05$ respectively). The body weight loss was very significant following a 6-week treatment of Sim or BBR (Table 2). Besides, as shown in Table 2, the 6-week HFD regimen significantly increased serum TG, TC, LDL-C and reduced serum HDL-C levels in HFD rats compared with control rats $(\mathrm{P}<0.05$ and $\mathrm{P}<0.01$ respectively). However, a 6 -week treatment of BBR $(400 \mathrm{mg} / \mathrm{kg} / \mathrm{d})$ and $\operatorname{Sim}(2 \mathrm{mg} / \mathrm{kg} / \mathrm{d})$ significantly decreased the serum levels of TG $(\mathrm{P}<0.05$ and $\mathrm{P}<0.01$ respectively). Meanwhile, Sim significantly decreased serum TC and LDL-C $(\mathrm{P}<0.05)$. Both Sim and BBR could increase the serum levels of HDL-C, while BBR was more prominent in decreasing serum TG concentration and increasing HDL-C concentration compared with Sim group $(\mathrm{P}<0.01$ respectively).

\section{Elevation of plasma PCSK9 levels}

As shown in Figure 1, plasma PCSK9 levels were significantly higher in rats fed with HFD compared to that in rats fed with normal diet $(\mathrm{P}<0.05)$, suggesting that the HFD could up-regulate the expression of PCSK9. More interesting, rats treated by BBR showed a higher plasma 
Table 2 Changes of body weight and plasma lipid profile

\begin{tabular}{lcccc}
\hline & Con & HFD & HFD + Sim & HFD + BBR \\
\hline & $(\mathrm{n}=8)$ & $(\mathrm{n}=8)$ & $(\mathrm{n}=8)$ & $(\mathrm{n}=8)$ \\
Body weight $(\mathrm{g})$ & $325 \pm 5.8$ & $405 \pm 4.4^{*}$ & $345 \pm 9.4^{\#}$ & $360 \pm 8.7^{\#}$ \\
TC $(\mathrm{mmol} / \mathrm{L})$ & $2.20 \pm 0.07$ & $2.33 \pm 0.12$ & $2.10 \pm 0.12^{\#}$ & $2.28 \pm 0.12$ \\
TG $(\mathrm{mmo} / \mathrm{L})$ & $0.70 \pm 0.008$ & $1.80 \pm 0.003^{*}$ & $1.20 \pm 0.015^{\#}$ & $0.80 \pm 0.019^{\# \#}$ \\
LDL-C $(\mathrm{mmol} / \mathrm{L})$ & $0.46 \pm 0.02$ & $0.93 \pm 0.03^{*}$ & $0.56 \pm 0.04^{\#}$ & $0.52 \pm 0.04^{\#}$ \\
HDL-C $(\mathrm{mmol} / \mathrm{L})$ & $1.49 \pm 0.04$ & $1.10 \pm 0.03$ & $1.32 \pm 0.10$ & $1.60 \pm 0.02^{\# \#}$ \\
\hline
\end{tabular}

Con = control; HFD = high fat diet; HFD + Sim = high fat diet + simvastatin; HFD + BBR = high fat diet + berberine; TC = total cholesterol; TG = triglyceride; LDL-C = low density lipoprotein cholesterol; HDL-C = high density lipoprotein cholesterol. ${ }^{*}<0.05$ vs Con; ${ }^{\#}<0.05$ vs HFD; ${ }^{\# \#}<0.01$ vs HFD.

PCSK9 levels, which was similar to that treated by Sim compared with rat fed with HFD alone $(\mathrm{P}<0.05)$.

\section{mRNA expression of hepatic LDLR, SREBP-2 and HNF1}

As presented in Figure 2, Sim and BBR treatment for 6 weeks significantly increased the levels of hepatic LDLR and SREBP-2 mRNA in rats fed with HFD compared with that in rat fed with HFD alone $(\mathrm{P}<0.05)$. Meanwhile, Sim and BBR treatment remarked decreased the levels of hepatic HNF1 mRNA in rats fed with HFD compared with that in rat fed with HFD alone $(\mathrm{P}<0.05)$.

\section{Protein expression of hepatic LDLR, SREBP-2}

The pattern of protein expression of hepatic LDLR, SREBP-2 was very similar to the mRNA expression in our present study. As summarized in Figure 3, Sim and BBR treatment for 6 weeks also significantly increased the expression of hepatic LDLR and SREBP-2 protein in rats fed with HFD compared with that in rat fed with HFD alone $(\mathrm{P}<0.01)$.

\section{Discussion}

The main findings of the present study are: (1) Sim and BBR decreased body weight and improved plasma lipid profile in HFD rats; (2) Sim and BBR increased circulating PCSK9 levels in HFD rats; (3) Sim and BBR increased mRNA levels of LDLR and SREBP-2 but decreased mRNA levels of HNF1 in the liver of HFD rats. (4) Changes of mRNA levels of LDLR and SREBP-2 in the liver of HFD rats were in consistent with that at protein levels, suggesting that enhanced circulating PCSK9 concentration by berberine was associated with SREBP-2 pathway in high fat diet-fed rats. Thus, the present study provided novel information regarding the impact of BBR on HFD-induced obesity and lipid disorders in vivo.

Obesity and overweight have been shown to be associated with cardiovascular diseases (CVD) such as arteriosclerosis, stroke and myocardial infarction, which were closely associated with lipoprotein metabolism [22]. It has been well recognized that the body weight gain can elevate TC, TG and LDL-C levels and reduce HDL-C

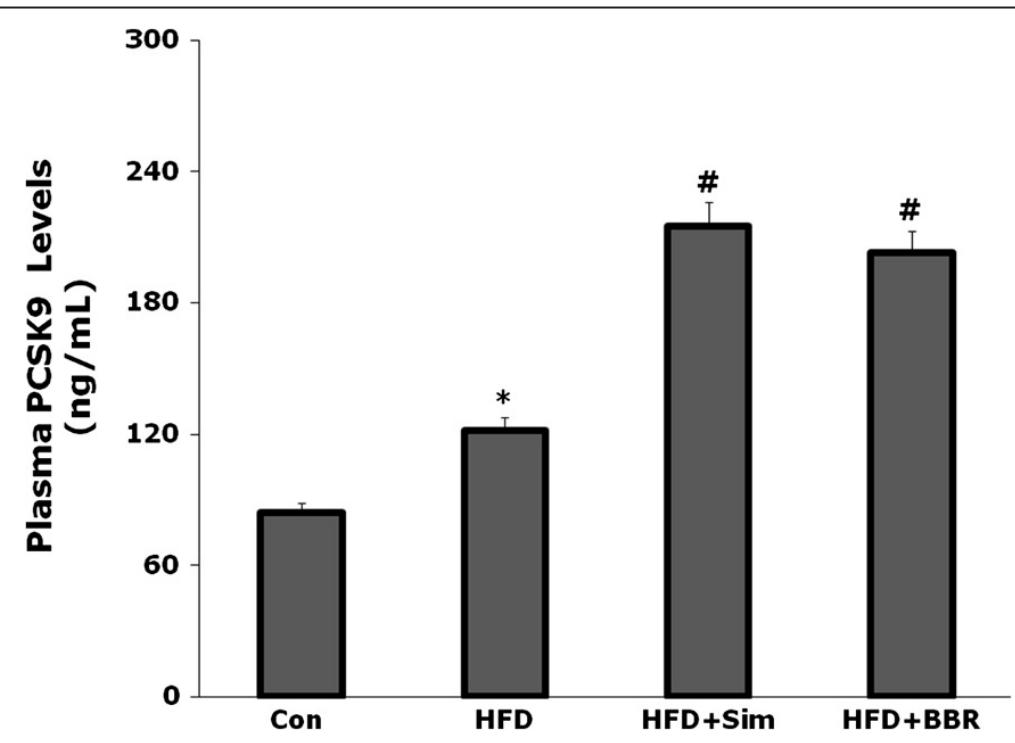

Figure 1 Effects of simvastatin and berberine on plasma PCSK9 concentration in rats at 6 weeks assessed by ELISA. Values are mean \pm SD, $n=8$. ${ }^{*} \mathrm{P}<0.05$, HFD vs Con; ${ }^{\#} \mathrm{P}<0.05, \mathrm{HFD}+\mathrm{BBR}$ or HFD + Sim vs HFD. Con = control; HFD = high fat diet; HFD + BBR = HFD + berberine; $\mathrm{HFD}+\mathrm{Sim}=\mathrm{HFD}+$ simvastatin. 


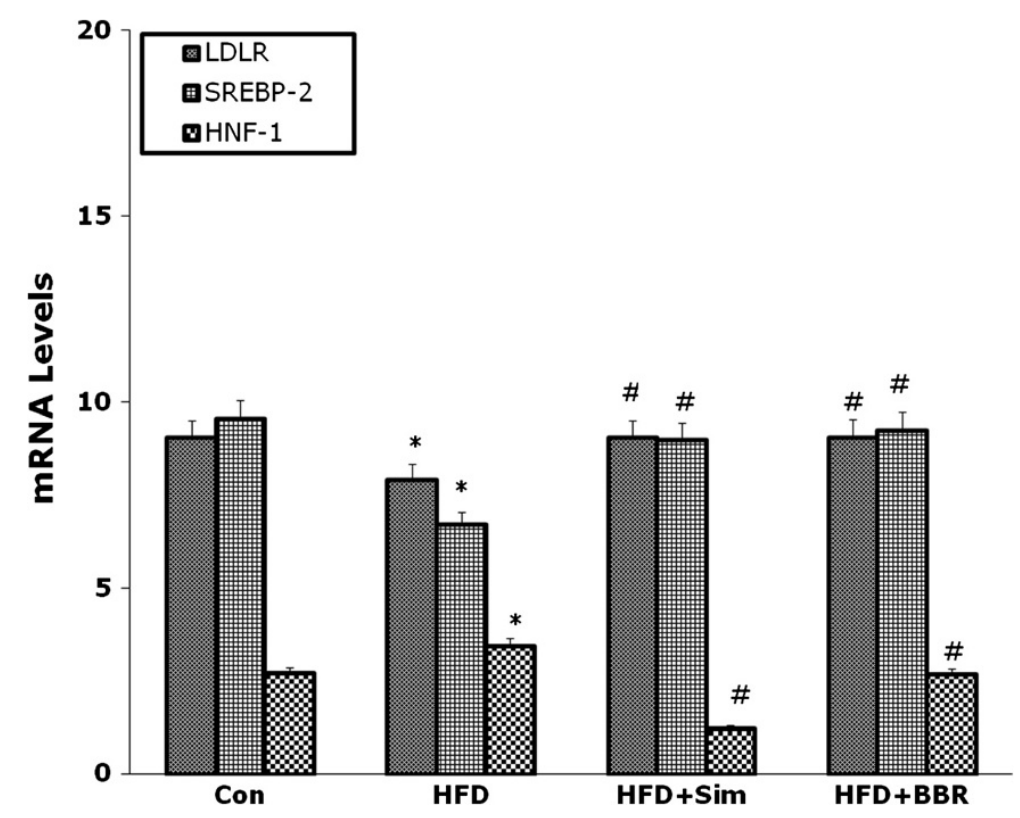

Figure 2 Effects of simvastatin and BBR on LDLR, SREBP-2 and HNF1 mRNA expression in the liver tissues at 6 weeks assessed by RT-PCR. Data were normalized to the GAPDH mRNA levels and then compared to control group or HFD group measurements. Values are mean \pm SD, $\mathrm{n}=3$. ${ }^{*} \mathrm{P}<0.05$, HFD vs Con; $\# \mathrm{P}<0.05, \mathrm{HFD}+\mathrm{Sim}$ or HFD + BBR vs HFD. Con = control; HFD = high fat diet; HFD + BBR = HFD + berberine; HFD + Sim = HFD + simvastatin; LDLR = low density lipoprotein receptor; SREBP-2 = sterol regulatory element binding protein-2; HNF1 = Hepatocyte nuclear factor 1 ; RT-PCR $=$ Real time quantitative reverse polymerase chain reaction.

level. Conversely, the weight loss is associated with lower levels of TG and LDL-C and higher levels of HDL-C, which can confer to cardiovascular benefit. Thus, the control of body weight is very important to affect cardiovascular health [23]. In the present study, the data indicated that BBR and Sim could significantly reduce the body weight and improve the lipid profile in rats fed with HFD compared with that in rats fed with HFD alone $(\mathrm{P}<0.05)$.

During the past few years, it has become clear that the major classes of usually prescribed lipid-lowering drugs such as statins elevated circulating PCSK9 levels $[14,15]$. Moreover, previous studies suggested that PCSK9 and LDLR both contain an SRE motif in their proximal promoters and thus are coordinately up-regulated by statins through activation of SREBP [13-16,24,25]. Alborn et al. found that there was a strong correlation of LDL-C and PSCK9 levels in humans [26]. Several studies have demonstrated the induction of PCSK9 by statins in cultured cells and in animal models [26]. In human studies, Careskey and colleagues firstly in 2008 reported that atorvastatin could increase circulating PCSK9 levels by the activation or nuclear translocation of SREBP-2, a transcription factor that activates both the LDLR and PCSK9 genes [18]. Subsequently, the impact of lipidlowering drugs on plasma PCSK9 concentration has intensively been investigated [14-17,20-33]. This effect of Sim on plasma PCSK9 was also identified in our present animal study. Thus, findings from in vitro and in vivo studies raised an important question whether the PCSK9 gene transcription could be separately regulated from LDLR.

It has been demonstrated that BBR improved lipid profile by post-transcriptional mechanism, which involves the activation of the extracellular signal-regulated kinase (ERK) signaling pathway [30]. In recent years, the studies have also indicated that BBR can down-regulate the transcription of PCSK9 while up-regulate LDLR mRNA level post-transcriptionally in HepG2 cells [20,30]. This investigation led us to uncover a novel transcriptionally regulatory role that modulated the expression of PCSK9 in vivo. In the present study, data showed, for the first time, that the mRNA levels of PCSK9, LDLR and SREBP-2 of the livers in HFD rats were significantly up-regulated while that of HNF1 was down-regulated by BBR, which was also demonstrated at protein levels assessed by western blotting analysis.

PCSK9, a serine protease expresses in the liver and intestine, which moves to the cell surface after autocleavage in endoplasmic reticulum (ER) and is secreted into the plasma [31]. The role of PCSK9 as post-transcriptional regulator of the amount of LDLRs in the liver is supported by the finding that gain of function mutations of PCSK9 in humans could cause hyperocholesterolemia, resulting in a increase of cardiovascular risk [31]. It has been indicated that the promoter region of PCSK9 gene contains the 


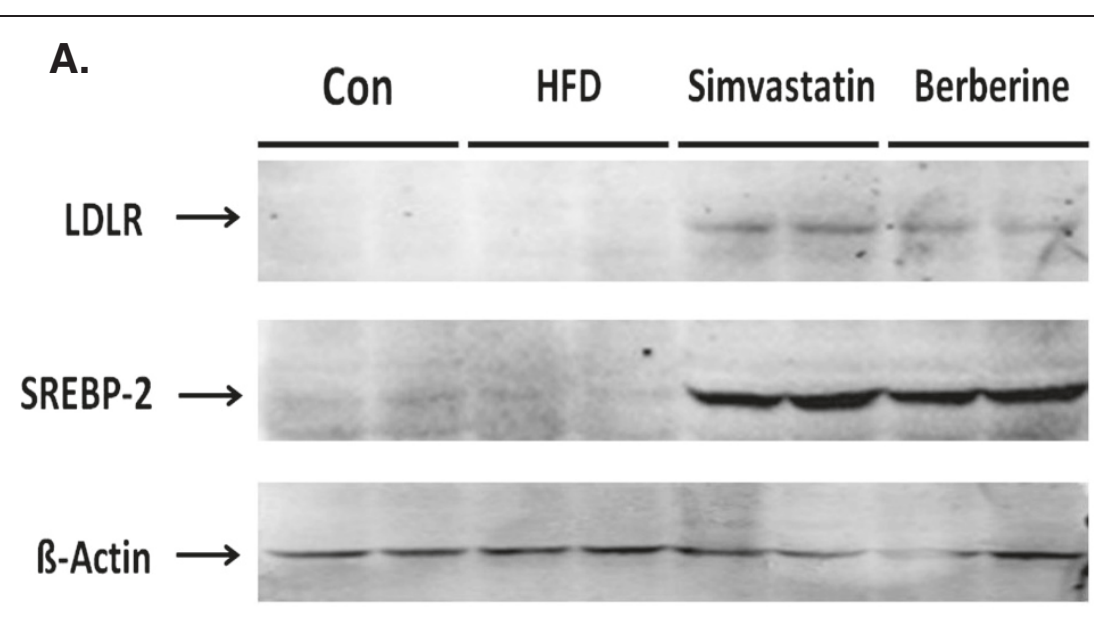

B.

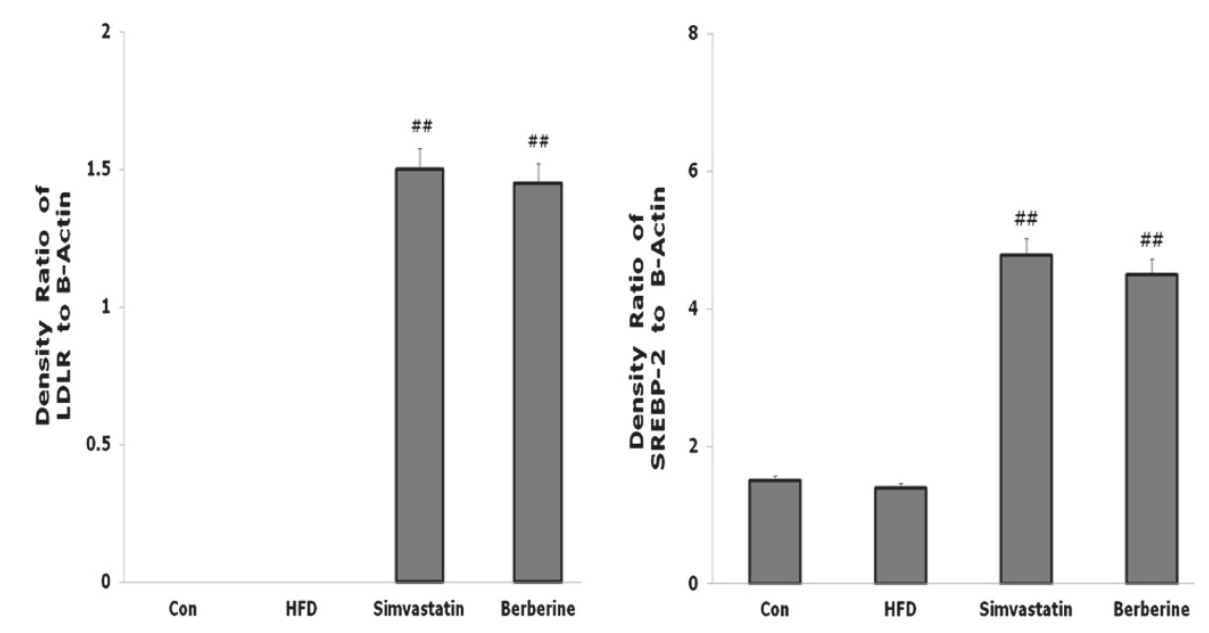

Figure 3 Effects of simvastatin and BBR on LDLR and SREBP-2 protein expression of the liver tissues at 6 weeks assessed by western blotting analysis. A. A representative western blot analysis is shown; $\mathbf{B}$. Western blots were quantitated as described in methods. Con = control; $\mathrm{HFD}=$ high fat diet; Simvastatin = HFD + simvastatin; Berberine = HFD + berberine; LDLR = low density lipoprotein receptor; SREBP-2= sterol regulatory element binding protein-2.

HNF1 and SRE sites, which respectively bind the transactivation proteins HNF1 and SREBP2. HNF1 is essential for PCSK9 basal transcription and for SREBP2- induced maximal gene expression in response to intracellular cholesterol depletion [32].

In the present study, in order to elucidating the effects of BBR on expression of PCSK9, we provide new evidence to support the notion that lipid drugs increase of PCSK9 and LDLR expression in the process of lipidlowering treatment. This led us to speculate how BBR affects expression of PCSK9 gene and what interactions of both SREBP and HNF1 factors are involved. By RTPCR analysis we first showed that the expression of PCSK9 was higher in BBR- and Sim-treated rats compared with that in rat fed HFD alone $(\mathrm{P}<0.05)$. Meanwhile, the enhanced expression of LDLR and SREBP-2 were also found in BBR-treated and Sim-treated rats compared that with HFD rat alone $(\mathrm{P}<0.05)$. In contrast, the expression of HNF1 was lower in BBR-treated and Sim-treated rats compared with that in HFD rats $(\mathrm{P}<0.05)$. The data imply an effect occurred in BBRtreated rats by down-regulation of HNF1 transcription factor at modest levels. This observed would be beneficial with regard to LDLR expression because SREBP2 is absolutely required for LDLR transcription, and a strong improved expression of SREBP2 would eventually increased LDLR expression. The facts that BBR treatment increases LDLR protein level in HFD rats suggested that the balanced effects of BBR are in favor of LDLR expression and stability. In additional, BBR-treatment increased the expression of PCSK9 similar to Sim in HFD rats. Interestingly, for the first time, we showed that BBR also 
up-regulated PCSK9, LDLR and SREBP-2 in HFD rats. Genevieve's study found that the regulation of hepatic LDLR expression by statins is multifactorial including transcriptional activation through SREBP-2 and posttranscriptional modulational modulations by PCSK9 $[16,26]$. It is possible, statin increased hepatic LDLRs that would act to bind the PCSK9 in the circulation. Further increased expression of PCSK9 protein might exceed LDLR binding, resulting in increased circulating levels of PCSK9 protein and only a modest additional decrease in LDL-C [17]. LDLR and SREBP-2 mRNA up-regulation translates into increased circulating PCSK9 protein, adding a PCSK9 inhibitor to statin therapy presents the possibility of further lowering LDLC to recommended concentrations in patients unable to attain desired LDLC on statin therapy alone [34]. Thus, our study confirmed previous studies and provided novel additional important information regarding the lipid-lowering drugs on plasma PCSK9. Given that statins and BBR improved lipid metabolism levels in HFD and increase PCSK9 at the same time, it is reasonable to infer that a combination of BBR and an inhibitor of PCSK9, such as monoclonal antibody targeting PCSK9, would bring greater lipid-lowering effects in the future.

\section{Conclusion}

The present study demonstrated that BBR could reduce the body weight and improve the blood lipid profile of rat fed with HFD through up-regulating the expression of SREBP-2, suggesting that impacts of BBR on lipid profile might also be linked to SREBP-2 pathway. Further studies are required to determine the clinical significance involved in the up-regulation of PCSK9 by BBR.

\section{Competing interests}

The authors declare that they have no competing interests.

\section{Authors' contributions \\ YJJ carried out the animal study, analyzed the data, and drafted the manuscript. RXX and JS participated in the measurement and analysis of plasma PCSK9 and gene expressions. JJL and YT conceived and designed the study, supervised all the experiments, interpreted the data, and edited the manuscript. All authors read and approved the final manuscript.}

\section{Acknowledgements}

This article is partly supported by National Natural Scientific Foundation (81070171, 81241121), Specialized Research Fund for the Doctoral Program of Higher Education of China (20111106110013), Capital Special Foundation of Clinical Application Research (Z121107001012015), Capital Health Development Fund (2011400302), and Beijing Natural Scientific Foundation (7131014) awarded to Dr. Jian-Jun Li, MD, PhD.

\section{Received: 28 January 2014 Accepted: 14 April 2014}

Published: 23 April 2014

\section{References}

1. Liao FH, Shieh MJ, Yang SC, Lin SH, Chien YW: Effectiveness of a soy-based compared with a traditional low-calorie diet on weight loss and lipid levels in overweight adults. Nutrition 2007, 23:551-556.

2. Eckel RH, Grundy SM, Zimmet PZ: The metabolic syndrome. Lancet 2005, 365:1415-1428.
3. Brown MS, Goldstein JL: Receptor-mediated endocytosis: insights from the lipoprotein receptor system. Proc Natl Acad Sci U S A 1979, 76:3330-3337.

4. Cariou B, Le May C, Costet P: Clinical aspects of PCSK9. Atherosclerosis 2011, 216:258-265.

5. Kingsley DM, Krieger M: Receptor-mediated endocytosis of low density lipoprotein: somatic cell mutants define multiple genes required for expression of surface-receptor activity. Proc Natl Acad Sci U S A 1984, 81:5454-5458.

6. Zhang DW, Lagace TA, Garuti R, Zhao Z, McDonald M, Horton JD, Cohen JC, Hobbs HH: Binding of proprotein convertase subtilisin/kexin type 9 to epidermal growth factor-like repeat $A$ of low density lipoprotein receptor decreases receptor recycling and increases degradation. J Biol Chem 2007, 282:18602-18612.

7. McNutt MC, Kwon HJ, Chen C, Chen JR, Horton JD, Lagace TA: Antagonism of secreted PCSK9 increases low density lipoprotein receptor expression in HepG2 cells. J Biol Chem 2009, 284:10561-10570.

8. Li J, Tumanut C, Gavigan JA, Huang WJ, Hampton EN, Tumanut R, Suen KF, Trauger JW, Spraggon G, Lesley SA, Liau G, Yowe D, Harris JL: Secreted PCSK9 promotes LDL receptor degradation independently of proteolytic activity. Biochem J 2007, 406:203-207.

9. Brown MS, Goldstein JL: The SREBP pathway: regulation of cholesterol metabolism by proteolysis of a membrane-bound transcription factor. Cell 1997, 89:331-340.

10. Brown MS, Goldstein JL: A proteolytic pathway that controls the cholesterol content of membranes, cells, and blood. Proc Natl Acad Sci U S A 1999, 96:11041-11048

11. Horton JD, Goldstein JL, Brown MS: SREBPs: activators of the complete program of cholesterol and fatty acid synthesis in the liver. J Clin Invest 2002, 109:1125-1131.

12. Attie AD, Seidah NG: Dual regulation of the LDL receptor-some clarity and new questions. Cell Metab 2005, 1:290-292.

13. Stancu C, Sima A: Statins: mechanism of action and effects. J Cell Mol Med 2001, 5:378-387.

14. Guo YL, Liu J, Xu RX, Zhu CG, Wu NQ, Jiang LX, Li JJ: Short-term impact of low-dose atorvastatin on serum proprotein convertase subtilisin/kexin type 9. Clin Drug Investig 2013, 33:877-883.

15. Liu J, Guo YL, Xu RX, Li JJ: Rapid effects of different lipid-lowering drugs on PCSK9 in humans. Clin Lipidol 2013, 8:519-524.

16. Dubuc G, Chamberland A, Wassef H, Davignon J, Seidah NG, Bernier L, Prat A: Statins upregulate PCSK9, the gene encoding the proprotein convertase neural apoptosis-regulated convertase-1 implicated in familial hypercholesterolemia. Arterioscler Thromb Vasc Biol 2004, 24:1454-1459.

17. Careskey HE, Davis RA, Alborn WE, Troutt JS, Cao GQ, Konrad RJ: Atorvastatin increases human serum levels of proprotein convertase subtilisin/kexin type 9. J Lipid Res 2008, 49:394-398.

18. Goldstein JL, Brown MS, Anderson RG, Russell DW, Schneider WJ: Receptormediated endocytosis: concepts emerging from the LDL receptor system. Annu Rev Cell Biol 1985, 1:1-39.

19. Jeong HJ, Lee HS, Kim KS, Kim YK, Yoon D, Park SW: Sterol-dependent regulation of proprotein convertase subtilisin/kexin type 9 expression by sterol-regulatory element binding protein-2. J Lipid Res 2008, 49:399-409.

20. Choi BH, Ahn IS, Kim YH, Park JW, Lee SY, Hyun CK, Do MS: Berberine reduces the expression of adipogenic enzymes and inflammatory molecules of 3 T3-L1 adipocyte. Exp Mol Med 2006, 38:599-605.

21. Kong W, Wei J, Abidi P, Lin M, Inaba S, Li C, Wang Y, Wang Z, Si S, Pan H, Wang S, Wu J, Wang Y, Li Z, Liu J, Jiang JD: Berberine is a novel cholesterol-lowering drug working through a unique mechanism distinct from statins. Nat Med 2004, 10:1344-1351

22. Lee YS, Kim WS, Kim KH, Yoon MJ, Cho HJ, Shen Y, Ye JM, Lee CH, Oh WK, Kim CT, Hohnen-Behrens C, Gosby A, Kraegen EW, James DE, Kim JB: Berberine, a natural plant product, activates AMP-activated protein kinase with beneficial metabolic effects in diabetic and insulin-resistant states. Diabetes 2006, 55:2256-2264.

23. Hu Y, Davies GE: Berberine inhibits adipogenesis in high-fat diet-induced obesity mice. Fitoterapia 2010, 81:358-366.

24. Cabioglu MT, Ergene N: Electroacupuncture therapy for weight loss reduces serum total cholesterol, triglycerides, and LDL cholesterol levels in obese women. Am J Chin Med 2005, 33:525-533.

25. Wang Y, Huang Y, Hobbs HH, Cohen JC: Molecular characterization of proprotein convertase subtilisin/kexin type 9-mediated degradation of the LDLR. J Lipid Res 2012, 53:1932-1943. 
26. Alborn WE, Cao G, Careskey HE, Qian YW, Subramaniam DR, Davies J, Conner EM, Konrad RJ: Serum proprotein convertase subtilisin kexin type 9 is correlated with serum LDL cholesterol. Clin Chem 2007, 53:1814-1819.

27. Briggs MR, Yokoyama C, Wang X, Brown MS, Goldstein JL: Nuclear protein that binds sterol regulatory element of low density lipoprotein receptor promoter. I. Identification of the protein and delineation of its target nucleotide sequence. J Biol Chem 1993, 268:14490-14496.

28. Misawa K, Horiba T, Arimura N, Hirano Y, Inoue J, Emoto N, Shimano H, Shimizu M, Sato R: Sterol regulatory element-binding protein-2 interacts with hepatocyte nuclear factor-4 to enhance sterol isomerase gene expression in hepatocytes. J Biol Chem 2003, 278:36176-36182.

29. Dong B, Wu M, Li H, Kraemer FB, Adeli K, Seidah NG, Park SW, Liu J: Strong induction of PCSK9 gene expression through HNF1alpha and SREBP2: mechanism for the resistance to LDL-cholesterol lowering effect of statins in dyslipidemic hamsters. J Lipid Res 2010, 51:1486-1495.

30. Welder G, Zineh I, Pacanowski MA, Troutt JS, Cao G, Konrad RJ: High-dose atorvastatin causes a rapid sustained increase in human serum PCSK9 and disrupts its correlation with LDL cholesterol. J Lipid Res 2010, 51:2714-2721

31. Li H, Chen W, Zhou Y, Abidi P, Sharpe O, Robinson WH, Kraemer FB, Liu J: Identification of mRNA binding proteins that regulate the stability of LDL receptor mRNA through AU-rich elements. J Lipid Res 2009, 50:820-831.

32. Cameron J, Ranheim T, Kulseth MA, Leren TP, Berge KE: Berberine decreases PCSK9 expression in HepG2 cells. Atherosclerosis 2008, 201:266-273.

33. Zhou Y, Cao S, Wang Y, Xu P, Yan J, Bin W, Qiu F, Kang N: Berberine metabolites could induce low density lipoprotein receptor up-regulation to exert lipid-lowering effects in human hepatoma cells. Fitoterapia 2014, 92:230-237.

34. Li H, Dong B, Park SW, Lee HS, Chen W, Liu J: Hepatocyte nuclear factor 1alpha plays a critical role in PCSK9 gene transcription and regulation by the natural hypocholesterolemic compound berberine. J Biol Chem 2009, 284:28885-28895.

doi:10.1186/1479-5876-12-103

Cite this article as: Jia et al.: Enhanced circulating PCSK9 concentration by berberine through SREBP-2 pathway in high fat diet-fed rats. Journal of Translational Medicine 2014 12:103.

\section{Submit your next manuscript to BioMed Central and take full advantage of:}

- Convenient online submission

- Thorough peer review

- No space constraints or color figure charges

- Immediate publication on acceptance

- Inclusion in PubMed, CAS, Scopus and Google Scholar

- Research which is freely available for redistribution 